\title{
On Degree-Based Topological Indices for Strong Double Graphs
}

\author{
Muhammad Rafiullah $\mathbb{D}^{1}{ }^{1}$ Hafiz Muhammad Afzal Siddiqui $\mathbb{D}^{1}$ \\ Muhammad Kamran Siddiqui, ${ }^{1}$ and Mlamuli Dhlamini $\mathbb{D D}^{2}$ \\ ${ }^{1}$ Department of Mathematics, COMSATS University Islamabad Lahore Campus, Lahore 54000, Pakistan \\ ${ }^{2}$ Department of Applied Mathematics, National University of Science and Technology, Bulawayo, Zimbabwe
}

Correspondence should be addressed to Mlamuli Dhlamini; mlamuli.dhlamini@nust.ac.zw

Received 10 June 2021; Accepted 21 July 2021; Published 2 August 2021

Academic Editor: Syed Ahtsham Haq Bokhary

Copyright $(\odot 2021$ Muhammad Rafiullah et al. This is an open access article distributed under the Creative Commons Attribution License, which permits unrestricted use, distribution, and reproduction in any medium, provided the original work is properly cited.

A topological index is a characteristic value which represents some structural properties of a chemical graph. We study strong double graphs and their generalization to compute Zagreb indices and Zagreb coindices. We provide their explicit computing formulas along with an algorithm to generate and verify the results. We also find the relation between these indices. A 3D graphical representation and graphs are also presented to understand the dynamics of the aforementioned topological indices.

\section{Introduction}

Chemical graph theory is an important topological field of mathematical chemistry that deals with mathematical modelling of chemical compound structures. A molecular structure of a compound consists of many atoms. Specially, hydrocarbons are chemical compounds which consist of carbon and hydrogen atoms. A graph consisting of hydrocarbons is known as a molecular graph which represents the carbon structure of a molecule [1].

We consider a simple molecular graph, say $G$, which consists of nonhydrogen atoms and covalent bonds. In graph theory, the nonhydrogen atoms are represented by a set of vertices $V=V(G)$ and the covalent bonds with the set of edges $E=E(G)$. The number of atoms and bonds in a structure is represented by $n=|V|$ and $m=|E|$, respectively. The valency of an atom is represented by $\mathfrak{R}_{G}(v)$, and it is known as the degree of vertex $v \in V(G)$, which also represents the number of adjacent (or neighboring) vertices of $V$. A set consisting of neighboring vertices of $v$ is known as open neighborhood and denoted as $N(v)=\{w \in V(G): v w \in E(G)\}$. If the vertex $v$ is included in open neighborhood, then the set of vertices is called closed neighborhood, and it is denoted as $N[v]=N(v) \cup\{v\}$.

A double graph $D(G)$ is generated by taking two copies of a graph $G$ and connecting every vertex $v_{i}$ in one copy with the opened neighborhood $N\left(v_{i}\right)$ of the corresponding vertex with its second copy. For details, see Figure 1 in which a path graph $P_{4}$ and its double graph are presented. A strong double graph $S D(G)$ is generated by taking two copies of a graph $G$ and connecting every vertex $v_{i}$ in one copy with closed neighborhood $N\left[v_{i}\right]=N\left(v_{i}\right) \cup\left\{v_{i}\right\}$ of the corresponding vertex with its second copy. For details, see Figure 2 in which a strong double graph of $P_{4}$ and its $k$-iterated strong double graph are presented.

A complement $\bar{G}$ of a graph $G$ consists of the same set of vertices, where two vertices $v$ and $w$ are adjacent by an edge $v w$ if and only if they are not adjacent in $G$. Hence, $v w \in E(\bar{G}) \Leftrightarrow v w \notin E(G)$. A complement of a graph consists of a number of edges and the degree of vertex $v$ which are represented as $\bar{m}=\left(\begin{array}{l}n \\ 2\end{array}\right)-m$ and $d_{\bar{G}}(v)=n-1-\mathfrak{R}_{G}(v)$, respectively.

A molecular descriptor is known as the topological index provides specific information about the structure of molecules. In graph theory, the molecular structure is considered as a graph $G$. The topological index is also known as the connectivity index [2,3]. Topological indices are largely applied in chemistry to develop the quantitative structureactivity relationship (QSAR) in which the characteristics of molecules can be correlated with their chemical structures [4]. The physicochemical properties of a molecule can also be explained through topological indices. The first index of a 


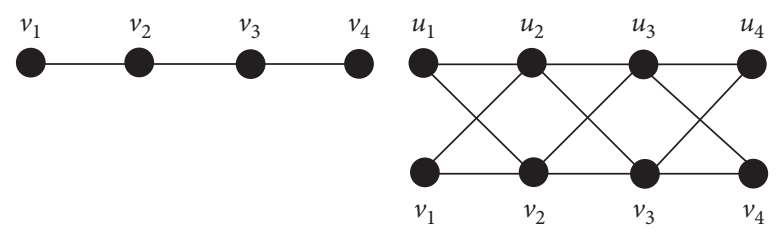

(a)

(b)

Figure 1: The graph $P_{4}$ and its double graph. (a) $P_{4}$. (b) $D\left(P_{4}\right)$.

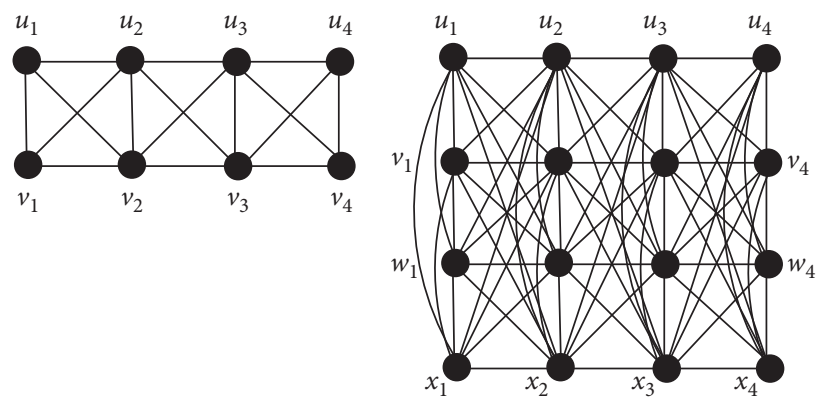

(a)

(b)

FIgURe 2: The strong double graph of $P_{4}$ and its generalization. (a) $S D^{* 1}\left(P_{4}\right)$. (b) $S D^{* 2}\left(P_{4}\right)$.

chemical graph was introduced by Harold Wiener [5] in 1947 as an aid to determining the boiling point of the paraffin compound. This index is known as the Wiener index and defined as $W(G)=\sum_{\{u, v\} \subseteq V(G)} d(u, v)$, where the notation $d(u, v)$ represents the distance between $u$ and $v$.

A topological index is defined as a function $T: \psi \longrightarrow \mathbb{R}$, where $\psi$ is the set of finite simple graphs and $\mathbb{R}$ is a set of real numbers which satisfy the relation $T(G)=T(H)$ if $G$ is isomorphic to $H$. Recently published work $[6,7]$ motivated us to further investigate the Zagreb indices and coindices of strong double graphs.

The first Zagreb index $M_{1}(G)$ and second Zagreb index $M_{2}(G)$ were introduced by Gutman and Trinajstić in 1972 [8] and elaborated by Nikolić et al. after 30 years in 2003 [9]. $M_{1}(G)$ and $M_{2}(G)$ are defined as

$$
\begin{aligned}
& M_{1}(G)=\sum_{u v \in E(G)}\left[\mathfrak{R}_{G}(u)+\mathfrak{R}_{G}(v)\right], \\
& M_{2}(G)=\sum_{u v \in E(G)}\left[\mathfrak{R}_{G}(u) \Re_{G}(v)\right] .
\end{aligned}
$$

The first Zagreb index is also written as $\sum_{v \in V(G)}\left[\Re_{G}(v)\right]^{2}$.

Recently, some useful versions of Zagreb indices have been discovered, such as multiplicative Zagreb indices $[3,7,10]$, multiplicative sum Zagreb indices [11, 12], Zagreb coindices [6], and multiplicative Zagreb coindices [13].

The important variants of the Zagreb index are the first and second Zagreb coindices, which are defined as follows, respectively:

$$
\begin{aligned}
& \bar{M}_{1}(G)=\sum_{u \neq v ; u v \notin E(G)}\left[\mathfrak{R}_{G}(u)+\mathfrak{R}_{G}(v)\right], \\
& \bar{M}_{2}(G)=\sum_{u \neq v ; u v \notin E(G)}\left[\mathfrak{R}_{G}(u) \mathfrak{R}_{G}(v)\right] .
\end{aligned}
$$

Doslic [14] introduced $\bar{M}_{1}(G)$ and $\bar{M}_{2}(G)$ in 2008. In 2009, Ashrafi et al. [15] determined the extremal values of these new invariants for some special graphs. They [6] also explored their fundamental properties and provided some explicit formulas for these versions under different graph operations.

\section{Main Results}

In this section, we study Zagreb indices and Zagreb coindices of strong double graphs. We also study these indices for generalized $k$-iterated strong double graphs. We use the concept of edge partition to reduce computation complexity and obtain computing formulas for these indices.

For the sake of simplicity, we consider $S D(G)=G^{1 *}=$ $G^{*}$ and $G^{k *}=\left(G^{(k-1) *}\right)^{*}$ for $k \geq 2$. Assume that $G^{0 *}=G$ for the sake of consistency.

In the following theorem, we study the first and second Zagreb indices of the strong double graph.

Theorem 1. Let $G$ be a simple connected graph of order $n$ and size $m$; then,

(i) $M_{1}\left(G^{*}\right)=8 M_{1}(G)+16 m+2 n$

(ii) $M_{2}\left(G^{*}\right)=16 M_{2}(G)+12 M_{1}(G)+12 m+n$

Proof. For the sake of convenience, we label all vertices in $G$ as $\left\{v_{1}, v_{2}, \ldots, v_{n}\right\}$. Suppose that $x_{i}$ and $y_{i}$ are the corresponding clone vertices, in strong double graph $G^{*}$, of $v_{i}$ for each $i=1, \ldots, n$.

For any given vertex $v_{i}$ in $G$ and its clone vertices $x_{i}$ and $y_{i}$, there exists $d_{G^{*}}\left(x_{i}\right)=d_{G^{*}}\left(y_{i}\right)=2 \mathfrak{R}_{G}\left(v_{i}\right)+1$ by the definition of the strong double graph. 
For $v_{i}, v_{j} \in V(G)$, if $v_{i} v_{j} \in E(G)$, then $x_{i} x_{j} \in E\left(G^{*}\right)$, $y_{i} y_{j} \in E\left(G^{*}\right), x_{i} y_{j} \in E\left(G^{*}\right)$, and $y_{i} x_{j} \in E\left(G^{*}\right)$.

So, we only need to consider the total contribution of the following three types of adjacent vertex pairs both to $M_{1}\left(G^{*}\right)$ and to $M_{2}\left(G^{*}\right)$.

Type 1: the adjacent vertex pairs $\left\{x_{i}, x_{j}\right\}$ and $\left\{y_{i}, y_{j}\right\}$, where $v_{i} v_{j} \in E(G)$
Type 2: the adjacent vertex pairs $\left\{x_{i}, y_{i}\right\}$ for each $i=1, \ldots, n$

Type 3: the adjacent vertex pairs $\left\{x_{i}, y_{j}\right\}$ and $\left\{y_{i}, x_{j}\right\}$, where $v_{i} v_{j} \in E(G)$

The total contribution of adjacent vertex pairs of type 1 in $M_{1}\left(G^{*}\right)$ is given by

$$
\begin{aligned}
\sum_{x_{i} x_{j} \in E\left(G^{*}\right)}\left[d_{G^{*}}\left(x_{i}\right)+d_{G^{*}}\left(x_{j}\right)\right] & =\sum_{y_{i} y_{j} \in E\left(G^{*}\right)}\left[d_{G^{*}}\left(y_{i}\right)+d_{G^{*}}\left(y_{j}\right)\right] \\
& =\sum_{v_{i} v_{j} \in E(G)}\left[\left(2 \mathfrak{R}_{G}\left(v_{i}\right)+1\right)+\left(2 \mathfrak{R}_{G}\left(v_{j}\right)+1\right)\right] \\
& =2 \sum_{v_{i} v_{j} \in E(G)}\left[\mathfrak{R}_{G}\left(v_{i}\right)+\mathfrak{R}_{G}\left(v_{j}\right)\right]+2|E(G)|_{v_{i} v_{j} \in E(G)} \\
& =2 M_{1}+2 m,
\end{aligned}
$$

and $M_{2}\left(G^{*}\right)$ is given by

$$
\begin{aligned}
\sum_{x_{i} x_{j} \in E\left(G^{*}\right)}\left[d_{G^{*}}\left(x_{i}\right) \cdot d_{G^{*}}\left(x_{j}\right)\right] & =\sum_{y_{i} y_{j} \in E\left(G^{*}\right)}\left[d_{G^{*}}\left(y_{i}\right) \cdot d_{G^{*}}\left(y_{j}\right)\right] \\
& =\sum_{v_{i} v_{j} \in E(G)}\left[\left(2 \mathfrak{R}_{G}\left(v_{i}\right)+1\right) \cdot\left(2 \mathfrak{R}_{G}\left(v_{j}\right)+1\right)\right] \\
& =4 \sum_{v_{i} v_{j} \in E(G)}\left[\mathfrak{R}_{G}\left(v_{i}\right) \cdot \mathfrak{R}_{G}\left(v_{j}\right)\right]+2 \sum_{v_{i} v_{j} \in E(G)}\left[\mathfrak{R}_{G}\left(v_{i}\right)+\mathfrak{R}_{G}\left(v_{j}\right)\right]+|E(G)|_{v_{i} v_{j} \in E(G)} \\
& =4 M_{2}+2 M_{1}+m .
\end{aligned}
$$

The total contribution of adjacent vertex pairs of type 2 in $M_{1}\left(G^{*}\right)$ is given by

$$
\begin{aligned}
& \sum_{x_{i} y_{i} \in E\left(G^{*}\right)}\left[d_{G^{*}}\left(x_{i}\right)+d_{G^{*}}\left(y_{i}\right)\right] \\
= & \sum_{v_{i} \in V(G)}\left[\left(2 \boldsymbol{R}_{G}\left(v_{i}\right)+1\right)+\left(2 \mathfrak{R}_{G}\left(v_{i}\right)+1\right)\right] \\
= & 4 \sum_{v_{i} \in V(G)} \boldsymbol{R}_{G}\left(v_{i}\right)+2|V(G)|_{v_{i} \in V(G)} \\
= & 8 m+2 n,
\end{aligned}
$$

and $M_{2}\left(G^{*}\right)$ is given by

$$
\begin{aligned}
& \sum_{x_{i} y_{i} \in E\left(G^{*}\right)}\left[d_{G^{*}}\left(x_{i}\right)+d_{G^{*}}\left(y_{i}\right)\right] \\
= & \sum_{v_{i} \in V(G)}\left[\left(2 \mathfrak{R}_{G}\left(v_{i}\right)+1\right) \cdot\left(2 \mathfrak{R}_{G}\left(v_{i}\right)+1\right)\right] \\
= & 4 \sum_{v_{i} \in V(G)}\left[\mathfrak{R}_{G}\left(v_{i}\right)\right]^{2}+4 \sum_{v_{i} \in V(G)} \mathfrak{R}_{G}\left(v_{i}\right)+|V(G)|_{v_{i} \in V(G)} \\
= & 4 M_{1}(G)+8 m+n .
\end{aligned}
$$

The total contribution of adjacent vertex pairs of type 3 in $M_{1}\left(G^{*}\right)$ is given by

$$
\begin{aligned}
\sum_{x_{i} y_{i} \in E\left(G^{*}\right)}\left[d_{G^{*}}\left(x_{i}\right)+d_{G^{*}}\left(y_{i}\right)\right] & =\sum_{y_{i} x_{j} \in E\left(G^{*}\right)}\left[d_{G^{*}}\left(y_{i}\right)+d_{G^{*}}\left(x_{i}\right)\right] \\
& =\sum_{v_{i} v_{j} \in E(G)}\left[\left(2 \mathfrak{R}_{G}\left(v_{i}\right)+1\right)+\left(2 \mathfrak{R}_{G}\left(v_{j}\right)+1\right)\right] \\
& =2 \sum_{v_{i} v_{j} \in E(G)}\left[\mathfrak{R}_{G}\left(v_{i}\right)+\mathfrak{R}_{G}\left(v_{i}\right)\right]+|E(G)|_{v_{i} v_{j} \in E(G)} \\
& =2 M_{1}(G)+2 m,
\end{aligned}
$$


and $M_{2}\left(G^{*}\right)$ is given by

$$
\begin{aligned}
\sum_{x_{i} y_{j} \in E\left(G^{*}\right)}\left[d_{G^{*}}\left(x_{i}\right) \cdot d_{G^{*}}\left(y_{j}\right)\right] & =\sum_{y_{i} x_{j} \in E\left(G^{*}\right)}\left[d_{G^{*}}\left(y_{i}\right) \cdot d_{G^{*}}\left(x_{j}\right)\right] \\
& =\sum_{v_{i} v_{j} \in E(G)}\left[\left(2 \mathfrak{R}_{G}\left(v_{i}\right)+1\right) \cdot\left(2 \mathfrak{R}_{G}\left(v_{j}\right)+1\right)\right] \\
& =4 \sum_{v_{i} v_{j} \in E(G)}\left[\mathfrak{R}_{G}\left(v_{i}\right) \cdot \mathfrak{R}_{G}\left(v_{j}\right)\right]+2 \sum_{v_{i} v_{j} \in E(G)}\left[\mathfrak{R}_{G}\left(v_{i}\right)+\mathfrak{R}_{G}\left(v_{j}\right)\right]+|E(G)|_{v_{i} v_{j} \in E(G)} \\
& =4 M_{2}(G)+2 M_{1}(G)+m .
\end{aligned}
$$

Therefore, by using equations (3), (5) and (7), we have

$$
\begin{aligned}
M_{1}\left(G^{*}\right)= & \sum_{x_{i} x_{j} \in E\left(G^{*}\right)}\left[d_{G^{*}}\left(x_{i}\right)+d_{G^{*}}\left(x_{j}\right)\right]+\sum_{y_{i} y_{j} \in E\left(G^{*}\right)}\left[d_{G^{*}}\left(y_{i}\right)+d_{G^{*}}\left(y_{i}\right)\right] \\
& +\sum_{x_{i} y_{i} \in E\left(G^{*}\right)}\left[d_{G^{*}}\left(x_{i}\right)+d_{G^{*}}\left(y_{i}\right)\right]+\sum_{x_{i} y_{j} \in E\left(G^{*}\right)}\left[d_{G^{*}}\left(x_{i}\right)+d_{G^{*}}\left(y_{i}\right)\right]+\sum_{y_{i} x_{j} \in E\left(G^{*}\right)}\left[d_{G^{*}}\left(y_{i}\right)+d_{G^{*}}\left(x_{j}\right)\right] \\
= & \left(2 M_{1}(G)+2 m\right)+\left(2 M_{1}(G)+2 m\right)+(8 m+2 n)+\left(2 M_{1}(G)+2 m\right)+\left(2 M_{1}(G)+2 m\right) \\
= & 8 M_{1}(G)+16 m+2 n .
\end{aligned}
$$

By using (4), (6) and (8), we also have

$$
\begin{aligned}
M_{2}\left(G^{*}\right)= & \sum_{x_{i} x_{j} \in E\left(G^{*}\right)}\left[d_{G^{*}}\left(x_{i}\right) \cdot d_{G^{*}}\left(x_{j}\right)\right]+\sum_{y_{i} y_{j} \in E\left(G^{*}\right)}\left[d_{G^{*}}\left(y_{i}\right) \cdot d_{G^{*}}\left(y_{i}\right)\right] \\
& +\sum_{x_{i} y_{i} \in E\left(G^{*}\right)}\left[d_{G^{*}}\left(x_{i}\right) \cdot d_{G^{*}}\left(y_{i}\right)\right]+\sum_{x_{i} y_{j} \in E\left(G^{*}\right)}\left[d_{G^{*}}\left(x_{i}\right) \cdot d_{G^{*}}\left(y_{i}\right)\right]+\sum_{y_{i} x_{j} \in E\left(G^{*}\right)}\left[d_{G^{*}}\left(y_{i}\right) \cdot d_{G^{*}}\left(x_{j}\right)\right] \\
= & \left(4 M_{2}(G)+2 M_{1}(G)+m\right)+\left(4 M_{2}(G)+2 M_{1}(G)+m\right) \\
& +\left(4 M_{1}(G)+8 m+n\right)+\left(4 M_{2}(G)+2 M_{1}(G)+m\right)+\left(4 M_{2}(G)+2 M_{1}(G)+m\right) \\
= & 16 M_{2}(G)+12 M_{1}(G)+12 m+n .
\end{aligned}
$$

In this theorem, we study the first and second Zagreb coindices of the strong double graph.

Theorem 2. Let $G$ be a simple connected graph of order $n$ and size $m$; then,

(i) $\bar{M}_{1}\left(G^{*}\right)=8 \bar{M}_{1}(G)+4 n^{2}-4 n-8 m$

(ii) $\bar{M}_{2}\left(G^{*}\right)=16 \bar{M}_{2}(G)+8 \bar{M}_{1}(G)+2 n^{2}-2 n-4 m$

Proof. For the sake of convenience, we label all vertices in $G$ as $\left\{v_{1}, v_{2}, \ldots, v_{n}\right\}$. Suppose that $x_{i}$ and $y_{i}$ are the corresponding clone vertices, in strong double graph $G^{*}$, of $v_{i}$ for each $i=1, \ldots, n$.
For any given vertex $v_{i}$ in $G$ and its clone vertices $x_{i}$ and $y_{i}$, there exists $d_{G^{*}}\left(x_{i}\right)=d_{G^{*}}\left(y_{i}\right)=2 \Re_{G}\left(v_{i}\right)+1$ by the definition of the strong double graph.

For $v_{i}, v_{j} \in V(G)$, if $v_{i} v_{j} \notin E(G)$, then $x_{i} x_{j} \notin E(G)$, $y_{i} y_{j} \notin E(G), x_{i} y_{j} \notin E(G)$, and $y_{i} x_{j} \notin E(G)$.

So, we only need to consider the total contribution of the following two types of nonadjacent vertex pairs both to $\bar{M}_{1}\left(G^{*}\right)$ and to $\bar{M}_{2}\left(G^{*}\right)$.

Type 1: the nonadjacent vertex pairs $\left\{x_{i}, x_{j}\right\}$ and $\left\{y_{i}, y_{j}\right\}$, where $v_{i} v_{j} \notin E(G)$

Type 2: the nonadjacent vertex pairs $\left\{x_{i}, y_{j}\right\}$ and $\left\{y_{i}, x_{j}\right\}$, where $v_{i} v_{j} \notin E(G)$

The total contribution of nonadjacent vertex pairs of type 1 in $\bar{M}_{1}\left(G^{*}\right)$ is given by 


$$
\begin{aligned}
\sum_{x_{i} x_{j} \notin E\left(G^{*}\right)}\left[d_{G^{*}}\left(x_{i}\right)+d_{G^{*}}\left(x_{j}\right)\right] & =\sum_{y_{i} y_{j} \notin E\left(G^{*}\right)}\left[d_{G^{*}}\left(y_{i}\right)+d_{G^{*}}\left(y_{j}\right)\right] \\
& =\sum_{v_{i} v_{j} \notin E(G)}\left[\left(2 \Re_{G}\left(v_{i}\right)+1\right)+\left(2 \Re_{G}\left(v_{j}\right)+1\right)\right] \\
& =2 \sum_{v_{i} v_{j} \notin E(G)}\left[\Re_{G}\left(v_{i}\right)+\Re_{G}\left(v_{j}\right)\right]+2|E(\bar{G})| \\
& =2 M_{1}(G)-2 m+n^{2}-n,
\end{aligned}
$$

and $\bar{M}_{2}\left(G^{*}\right)$ is given by

$$
\begin{aligned}
\sum_{x_{i} x_{j} \notin E\left(G^{*}\right)}\left[d_{G^{*}}\left(x_{i}\right) \cdot d_{G^{*}}\left(x_{j}\right)\right] & =\sum_{y_{i} y_{j} \notin E\left(G^{*}\right)}\left[d_{G^{*}}\left(y_{i}\right) \cdot d_{G^{*}}\left(y_{j}\right)\right] \\
& =\sum_{v_{i} v_{j} \notin E(G)}\left[\left(2 \mathfrak{R}_{G}\left(v_{i}\right)+1\right) \cdot\left(2 \mathfrak{R}_{G}\left(v_{j}\right)+1\right)\right] \\
& =4 \sum_{v_{i} v_{j} \notin E(G)}\left[\mathfrak{R}_{G}\left(v_{i}\right) \cdot \mathfrak{R}_{G}\left(v_{j}\right)\right]+2 \sum_{v_{i} v_{j} \notin E(G)}\left[\mathfrak{R}_{G}\left(v_{i}\right)+\mathfrak{R}_{G}\left(v_{j}\right)\right]+|E(\bar{G})| \\
& =4 M_{2}(G)+2 M_{1}(G)+\frac{1}{2}\left(n^{2}-n-2 m\right) .
\end{aligned}
$$

The total contribution of nonadjacent vertex pairs of type 2 in $\bar{M}_{1}\left(G^{*}\right)$ is given by

$$
\begin{aligned}
\sum_{x_{i} y_{j} \notin E\left(G^{*}\right)}\left[d_{G^{*}}\left(x_{i}\right)+d_{G^{*}}\left(y_{j}\right)\right] & =\sum_{y_{i} x_{j} \notin E\left(G^{*}\right)}\left[d_{G^{*}}\left(y_{i}\right)+d_{G^{*}}\left(x_{j}\right)\right] \\
& =\sum_{v_{i} v_{j} \notin E(G)}\left[\left(2 \mathfrak{R}_{G}\left(v_{i}\right)+1\right)+\left(2 \mathfrak{R}_{G}\left(v_{j}\right)+1\right)\right] \\
& =2 \sum_{v_{i} v_{j} \notin E(G)}\left[\mathfrak{R}_{G}\left(v_{i}\right)+\mathfrak{R}_{G}\left(v_{j}\right)\right]+2|E(\bar{G})| \\
& =2 M_{1}(G)-2 m+n^{2}-n,
\end{aligned}
$$

and $\bar{M}_{2}\left(G^{*}\right)$ is given by

$$
\begin{aligned}
\sum_{x_{i}, y_{j} \notin\left(G^{*}\right)}\left[d_{G^{*}}\left(x_{i}\right) \cdot d_{G^{*}}\left(y_{j}\right)\right] & =\sum_{y_{i}, x_{j} \notin E\left(G^{*}\right)}\left[d_{G^{*}}\left(x_{i}\right)+d_{G^{*}}\left(y_{j}\right)\right] \\
& =\sum_{v_{i}, v_{j} \notin E(G)}\left[\left(2 \mathfrak{R}_{G}\left(v_{i}\right)+1\right) \cdot\left(2 \mathfrak{R}_{G}\left(v_{j}\right)+1\right)\right] \\
& =4 \sum_{v_{i}, v_{j} \notin E(G)}\left[\Re_{G}\left(v_{i}\right) \cdot \mathfrak{R}_{G}\left(v_{j}\right)\right]+2 \sum_{v_{i}, v_{j} \notin E(G)} \mathfrak{R}_{G}\left(v_{i}\right)+\mathfrak{R}_{G}\left(v_{j}\right)+|E(\bar{G})| \\
& =4 M_{2}(G)+2 M_{1}(G)+\frac{1}{2}\left(n^{2}-n-2 m\right) .
\end{aligned}
$$


Therefore, by using equations (11) and (13), we have

$$
\begin{aligned}
\bar{M}_{1}\left(G^{*}\right)= & \sum_{x_{i}, x_{j} \notin E\left(G^{*}\right)}\left[d_{G^{*}}\left(x_{i}\right)+d_{G^{*}}\left(x_{j}\right)\right]=\sum_{y_{i}, y_{j} \notin E\left(G^{*}\right)}\left[d_{G^{*}}\left(y_{i}\right)+d_{G^{*}}\left(y_{j}\right)\right] \\
& +\sum_{x_{i} y_{j} \notin E\left(G^{*}\right)}\left[d_{G^{*}}\left(x_{i}\right)+d_{G^{*}}\left(y_{j}\right)\right]+\sum_{y_{i} x_{j} \notin E\left(G^{*}\right)} d_{G^{*}}\left(y_{i}\right)+d_{G^{*}}\left(x_{j}\right) \\
= & \left(2 M_{1}(G)-2 m+n^{2}-n\right)+\left(2 M_{1}(G)-2 m+n^{2}-n\right) \\
& +\left(2 M_{1}(G)-2 m+n^{2}-n\right)+\left(2 M_{1}(G)-2 m+n^{2}-n\right) \\
= & 8 M_{1}(G)-8 m+4 n^{2}-4 n .
\end{aligned}
$$

By using equations (12) and (14), we also have

$$
\begin{aligned}
\bar{M}_{2}\left(G^{*}\right)= & \sum_{x_{i}, x_{j} \notin E\left(G^{*}\right)}\left[d_{G^{*}}\left(x_{i}\right) \cdot d_{G^{*}}\left(x_{j}\right)\right]=\sum_{y_{i}, y_{j} \notin E\left(G^{*}\right)}\left[d_{G^{*}}\left(y_{i}\right) \cdot d_{G^{*}}\left(y_{j}\right)\right] \\
& +\sum_{x_{i}, y_{j} \notin E\left(G^{*}\right)}\left[d_{G^{*}}\left(x_{i}\right) \cdot d_{G^{*}}\left(y_{j}\right)\right]+\sum_{y_{i}, x_{j} \notin E\left(G^{*}\right)} d_{G^{*}}\left(y_{i}\right) \cdot d_{G^{*}}\left(x_{j}\right) \\
= & \left(4 M_{2}(G)+2 M_{1}(G)+\frac{1}{2}\left(n^{2}-n-2 m\right)\right)+\left(4 M_{2}(G)+2 M_{1}(G)+\frac{1}{2}\left(n^{2}-n-2 m\right)\right) \\
& +\left(4 M_{2}(G)+2 M_{1}(G)+\frac{1}{2}\left(n^{2}-n-2 m\right)\right)+\left(4 M_{2}(G)+2 M_{1}(G)+\frac{1}{2}\left(n^{2}-n-2 m\right)\right) \\
= & 16 M_{2}(G)+8 M_{1}(G)+2\left(n^{2}-n-2 m\right) .
\end{aligned}
$$

Now, we present the first and second indices and coindices of $k$-iterated strong double graphs.

Theorem 3. Let $G$ be a nontrivial graph of order $n$ and size $m$, and let $G^{k *}$ be its $k$-iterated strong double graph. Then,

$$
\begin{aligned}
\text { (i) } & M_{1}\left(G^{* k}\right)=8^{k} M_{1}(G)+2^{2(k+1)}\left(2^{k}-1\right) m+2^{k} \\
& \left(2^{k}-1\right)^{2} n \\
\text { (ii) } & M_{2}\left(G^{* k}\right)=3 \cdot 2^{3 k-1} \cdot\left(2^{k}-1\right) M_{1}(G)+4^{2 k} M_{2} \\
& (G)+3 \cdot 2^{2 k} \cdot\left(2^{k}-1\right)^{2} m+2^{k-1}\left(2^{k}-1\right)^{3} n \\
\text { (iii) } & \bar{M}_{1}\left(G^{* k}\right)=8^{k} \bar{M}_{1}(G)+\left(2^{k}-1\right) 4^{k}\left(n^{2}-n-2 m\right) \\
\text { (iv) } & \bar{M}_{2} \quad\left(G^{* k}\right)=2^{4 k} \bar{M}_{2}(G)+2^{3 k} \cdot\left(2^{k}-1\right) \bar{M}_{1}(G)+ \\
& 2^{2 k-1} \cdot\left(2^{k}-1\right)^{2}\left(n^{2}-n-2 m\right)
\end{aligned}
$$

Proof. For any nontrivial graph $G$ with $n$ vertices and $m$ edges, the number of vertices in $G^{*}$ is $2 n$ and the number of edges in $G^{*}$ is $2 m$ plus those edges between the sets $\left\{x_{1}, x_{2}, \ldots, x_{n}\right\}$ and $\left\{y_{1}, y_{2}, \ldots, y_{n}\right\}$, that is, $4^{k} m+n$.
Now, we deduce that $G^{k *}$ has $2^{k} m$ vertices and $4^{k} m+$ $\left(2^{2 k-1}-2^{k-1}\right) n$ edges.

(i) $M_{1}\left(G^{k *}\right)=8^{k} M_{1}(G)+2^{2(k+1)}$

$\left(2^{k}-1\right) m+2^{k}\left(2^{k}-1\right)^{2} n$.

As we know,

$$
M_{1}\left(G^{*}\right)=8 M_{1}(G)+16 m+2 n .
$$

Using the size of strong double graph $m=4^{k} m+\left(2^{2 k-1}-2^{k-1}\right) n$, we have

$$
\begin{aligned}
M_{1}\left(G^{*}\right) & =8 M_{1}(G)+16\left\{4^{k} m+\left(2^{2 k-1}-2^{k-1}\right) n\right\}+2 n \\
M_{1}\left(G^{k *}\right) & =8 M_{1}\left(G^{(k-1) *}\right)+4 \cdot 2^{2 k} m+\left(2^{2 k-1}-2^{k-1}+2\right) n .
\end{aligned}
$$

By Theorem 1 and the definition of the $k$-iterated strong double graph, for $k \geq 1$, we have 


$$
\begin{aligned}
M_{1}\left(G^{k *}\right)= & 8 M_{1}\left(G^{(k-1) *}\right)+4 \cdot 2^{2 k} m+\left(8 \cdot 2^{2 k-2}-8 \cdot 2^{k-1}+2^{k}\right) n \\
M_{1}\left(G^{k *}\right)= & 8\left\{8 M_{1}\left(G^{(k-2) *}\right)+4 \cdot 2^{2 k-2} m+\left(8 \cdot 2^{2 k-4}-8 \cdot 2^{k-2}+2^{k-1}\right) n\right\} \\
& +4 \cdot 2^{2 k} m+\left(8 \cdot 2^{2 k-2}-8 \cdot 2^{k-1}+2^{k}\right) n \\
= & 8^{2} M_{1}\left(G^{(k-2) *}\right)+12 \cdot 2^{2 k} m+2^{2 k-1}\left(6 \cdot 2^{2 k}-15 \cdot 2^{k}\right) n \\
= & 8^{2}\left\{8 M_{1}\left(G^{(k-3) *}\right)+4 \cdot 2^{2 k-4} m+\left(8 \cdot 2^{2 k-6}-8 \cdot 2^{k-3}+2^{k-2}\right) n\right\} \\
& +12 \cdot 2^{2 k} m+2^{2 k-1}\left(6 \cdot 2^{2 k}-15 \cdot 2^{k}\right) n \\
= & 8^{3} M_{1}\left(G^{(k-3) *}\right)+28 \cdot 2^{2 k} m+\left(14 \cdot 2^{2 k}-63 \cdot 2^{k}\right) n \\
M_{1}\left(G^{* k}\right)= & 8^{k} M_{1}(G)+2^{2(k+1)}\left(2^{k}-1\right) m+2^{k}\left(2^{k}-1\right)^{2} n .
\end{aligned}
$$

(ii) $M_{2}\left(G^{* k}\right)=3 \cdot 2^{3 k-1} \cdot\left(2^{k}-1\right) M_{1}$

$(G)+3 \cdot 2^{2 k} \cdot\left(2^{k}-1\right)^{2} m+2^{k-1}\left(2^{k}-1\right)^{3} n$.

As we know,

$M_{2}\left(G^{*}\right)=16 M_{2}(G)+12 M_{1}(G)+12 m+n$.
Using $\quad M_{1}\left(G^{*}\right)=8 M_{1}(G)+4 \cdot 2^{2 k} m+\left(2^{2 k-1}-\right.$ $\left.2^{k-1}+2\right) n$ and the number of edges of strong double graph $m=4^{k} m+\left(2^{2 k-1}-2^{k-1}\right) n$, we have

$$
\begin{aligned}
& M_{2}\left(G^{*}\right)=16 M_{2}(G)+12\left\{8 M_{1}(G)+4 \cdot 2^{2 k} m+\left(2^{2 k-1}-2^{k-1}+2\right) n\right\}+12\left\{4^{k} m+\left(2^{2 k-1}-2^{k-1}\right) n\right\}+n \\
& M_{2}\left(G^{*}\right)=16 M_{2}(G)+96 M_{1}(G)+\left(192+12 \cdot 2^{2 k}\right) m+\left(6 \cdot 2^{2 k}-6 \cdot 2^{k}+25\right) n
\end{aligned}
$$

By Theorem 1 and the definition of the $k$-iterated strong double graph, for $k \geq 1$, we have

$$
\begin{aligned}
& M_{2}\left(G^{*}\right)=16 M_{2}\left(G^{(k-1) *}\right)+12 \cdot 2^{3(k-1)} M_{1}(G)+\left(6 \cdot 2^{3(k-1)}+6 \cdot 2^{5(k-1)}\right) m+\left(6 \cdot 4^{k-1}-6 \cdot 2^{k-1}+5^{2(k-1)}\right) n \\
& =16\left\{16 M_{2}\left(G^{(k-2) *}\right)+12 \cdot 2^{3(k-2)} M_{1}(G)+\left(6 \cdot 2^{3(k-2)}+6 \cdot 2^{5(k-2)}\right) m\right\} \\
& +16 \cdot\left(6 \cdot 4^{k-2}-6 \cdot 2^{k-2}+5^{2(k-2)}\right) n+12 \cdot 2^{3(k-1)} M_{1}(G) \\
& +\left(6 \cdot 2^{3(k-1)}+6 \cdot 2^{5(k-1)}\right) m+\left(6 \cdot 4^{k-1}-6 \cdot 2^{k-1}+5^{2(k-1)}\right) n \\
& =16^{2} M_{2}\left(G^{(k-1) *}\right)+\frac{9 \cdot 2^{3(k)}}{2} M_{1}(G)+\left(\frac{9 \cdot 2^{3 k}}{4}+\frac{9 \cdot 2^{5 k}}{32}\right) m+\left(\frac{15 \cdot 2^{2 k}}{2}-\frac{41 \cdot 5^{2 k}}{625}+27 \cdot 2^{k}+1\right) n, \\
& M_{2}\left(G^{k *}\right)=16^{2}\left\{16 M_{2}\left(G^{(k-3) *}\right)+12 \cdot 2^{3(k-3)} M_{1}(G)+\left(6 \cdot 2^{3(k-3)}+6 \cdot 2^{5(k-3)}\right) m\right\} \\
& +16^{2} \cdot\left(6 \cdot 4^{k-3}-6 \cdot 2^{k-3}+5^{2(k-3)}\right) n+\frac{9 \cdot 2^{3(k)}}{2} M_{1}(G) \\
& +\left(\frac{9 \cdot 2^{3 k}}{4}+\frac{9 \cdot 2^{5 k}}{32}\right) m+\left(\frac{15 \cdot 2^{2 k}}{2}-\frac{41 \cdot 5^{2 k}}{625}+27 \cdot 2^{k}+1\right) n \\
& M_{2}\left(G^{*}\right)=16^{3} M_{2}\left(G^{(k-3) *}\right)+\frac{21 \cdot 2^{3 k}}{2} M_{1}(G)+\left(\frac{21 \cdot 2^{3 k}}{4}+\frac{21 \cdot 2^{5 k}}{64}\right) m+\left(\frac{63 \cdot 2^{2 k}}{2}+\frac{1281 \cdot 5^{2 k}}{15625}+219 \cdot 2^{k}+1\right) n \text {, } \\
& M_{2}\left(G^{* k}\right)=3 \cdot 2^{3 k-1} \cdot\left(2^{k}-1\right) M_{1}(G)+4^{2 k} M_{2}(G)+3 \cdot 2^{2 k} \cdot\left(2^{k}-1\right)^{2} m+2^{k-1}\left(2^{k}-1\right)^{3} n \text {. }
\end{aligned}
$$


(iii) $\bar{M}_{1}\left(G^{* k}\right)=8^{k} \bar{M}_{1}(G)+\left(2^{k}-1\right) 4^{k}\left(n^{2}-n-2 m\right)$.

$$
M_{1}\left(G^{*}\right)=8 \bar{M}_{1}\left(G^{*}\right)-8 m+4 n^{2}+4 n .
$$

By Theorem 1 and the definition of the $k$-iterated strong double graph, for $k \geq 1$, we have

$$
\begin{aligned}
M_{1}\left(G^{k *}\right)= & 8 \bar{M}_{1}\left(G^{(k-1) *}\right)-2^{2 k+1} m+2^{2 k} n^{2}+2^{2 k} n \\
M_{1}\left(G^{k *}\right)= & 8\left\{8 \bar{M}_{1}\left(G^{(k-2) *}\right)-2^{2(k-1)+1} m+2^{2(k-1)} n^{2}+2^{2(k-1)} n\right\} \\
& -2^{2 k+1} m+2^{2 k} n^{2}+2^{2 k} n \\
= & 8^{2} M_{1}\left(G^{(k-2) *}\right)-6 \cdot 2^{2 k} m+3 \cdot 2^{2 k} n^{2}+3 \cdot 2^{2 k} n \\
= & 8^{2}\left\{8 \bar{M}_{1}\left(G^{(k-3) *}\right)-2^{2(k-2)+1} m+2^{2(k-2)} n^{2}+2^{2(k-2)} n\right\}-6 \cdot 2^{2 k} m \\
& +3 \cdot 2^{2 k} n^{2}+3 \cdot 2^{2 k} n \\
= & 8^{3} \bar{M}_{1}\left(G^{(k-3) *}\right)-14 \cdot 2^{2 k} m+7 \cdot 2^{2 k} n^{2}+7 \cdot 2^{2 k} n \\
\vdots & \\
M_{1}\left(G^{k *}\right)= & 8^{k} \bar{M}_{1}(G)+\left(2^{k}-1\right) 4^{k}\left(n^{2}-n-2 m\right) .
\end{aligned}
$$

(iv) $\bar{M}_{2}\left(G^{* k}\right)=2^{4 k} \bar{M}_{2}(G)+2^{3 k} \cdot\left(2^{k}-1\right) \bar{M}_{1}(G)+2^{2 k-1}$. $\left(2^{k}-1\right)^{2}\left(n^{2}-n-2 m\right)$.

$\bar{M}_{2}\left(G^{*}\right)=16 \bar{M}_{2}(G)+8 \bar{M}_{1}(G)+2\left(n^{2}-n-2 m\right)$.

$$
\begin{aligned}
\bar{M}_{2}\left(G^{k *}\right)= & 16 \bar{M}_{2}\left(G^{(k-1) *}\right)+8 \bar{M}_{1}\left(G^{(k-1) *}\right) \\
& +2\left(n^{2}-n-2 m\right),
\end{aligned}
$$

and using $\bar{M}_{1}\left(G^{*}\right)=8 \bar{M}_{1}(G)-8 m+4 n^{2}+4 n$, we have

By Theorem 1 and the definition of the $k$-iterated strong double graph, for $k \geq 1$, we have

$$
\begin{aligned}
\bar{M}_{2}\left(G^{k *}\right) & =16 \bar{M}_{2}\left(G^{(k-1) *}\right)+8\left\{8 \bar{M}_{1}(G)-8 m+4 n^{2}+4 n\right\}+2\left(n^{2}-n-2 m\right) \\
& =16 \bar{M}_{2}\left(G^{(k-1) *}\right)+64 \bar{M}_{1}(G)+32\left(n^{2}-n-2 m\right)+2\left(n^{2}-n-2 m\right) .
\end{aligned}
$$

By rearranging the terms, we have 


$$
\begin{aligned}
& \bar{M}_{2}\left(G^{k *}\right)=16 \bar{M}_{2}\left(G^{(k-1) *}\right)+8 \cdot 8^{(k-1)} \bar{M}_{1}(G)+32^{(k-1)}\left(n^{2}-n-2 m\right) \\
& +2^{(3 k-3)}\left(n^{2}-n-2 m\right) \\
& =16\left\{16 \bar{M}_{2}\left(G^{(k-2) *}\right)+8 \cdot 8^{(k-2)} \bar{M}_{1}(G)+32^{(k-2)}\left(n^{2}-n-2 m\right)\right\} \\
& +16 \cdot 2^{(3 k-6)}\left(n^{2}-n-2 m\right)+8 \cdot 8^{(k-1)} \bar{M}_{1}(G) \\
& +32^{(k-1)}\left(n^{2}-n-2 m\right)+2^{(3 k-3)}\left(n^{2}-n-2 m\right) \\
& =16^{2} \bar{M}_{2}\left(G^{(k-2) *}\right)+3 \cdot 2^{(3 k)} \bar{M}_{1}(G)+\frac{3 \cdot 2^{(5 k)}}{64}\left(n^{2}-n-2 m\right) \\
& +\frac{3 \cdot 2^{(3 k)}}{8}\left(n^{2}-n-2 m\right) \\
& =16^{2}\left\{16 \bar{M}_{2}\left(G^{(k-3) *}\right)+8 \cdot 8^{(k-3)} \bar{M}_{1}(G)+32^{(k-3)}\left(n^{2}-n-2 m\right)\right\} \\
& +16^{2} \cdot 2^{(3 k-9)}\left(n^{2}-n-2 m\right)+3 \cdot 2^{(3 k)} \bar{M}_{1}(G) \\
& +\frac{3 \cdot 2^{(5 k)}}{64}\left(n^{2}-n-2 m\right)+\frac{3 \cdot 2^{(3 k)}}{8}\left(n^{2}-n-2 m\right) \\
& =16^{3} \bar{M}_{2}\left(G^{(k-3) *}\right)+7 \cdot 2^{(3 k)} \bar{M}_{1}(G)+\frac{7 \cdot 2^{(5 k)}}{128}\left(n^{2}-n-2 m\right) \\
& +\frac{7 \cdot 2^{(3 k)}}{8}\left(n^{2}-n-2 m\right) \\
& \vdots \\
& \bar{M}_{2}\left(G^{k *}\right)=2^{4 k} \bar{M}_{2}(G)+2^{3 k} \cdot\left(2^{k}-1\right) \bar{M}_{1}(G)+2^{2 k-1} \cdot\left(2^{k}-1\right)^{2}\left(n^{2}-n-2 m\right) .
\end{aligned}
$$

Here, we present an algorithm to generate and verify the first and second Zagreb indices and coindices of any finite simple connected graph $G$. This algorithm is based on the adjacency matrix of $G$ (Algorithm 1).

Example 1. Let $A, B$, and $C$ be the matrices of path graph $P_{4}$ and strong double graphs $S D^{1 *}\left(P_{4}\right)$ and $S D^{2 *}\left(P_{4}\right)$, respectively. These matrices are square and symmetric, and choose value 1 (one) at the $i$ th row and $j$ th column if $v_{i}$ and $v_{j}$ are adjacent vertices in a graph; otherwise, it is 0 . The suggested algorithm is applied on these matrices to compute the first Zagreb index $I M_{1}$, second Zagreb index $I M_{2}$, first Zagreb coindex $C M_{1}$, and second Zagreb coindex $C M_{2}$. These adjacency matrices have been obtained by drawing the respective graphs in "GraphTea" software; this software is easily available, and it is free of cost. 


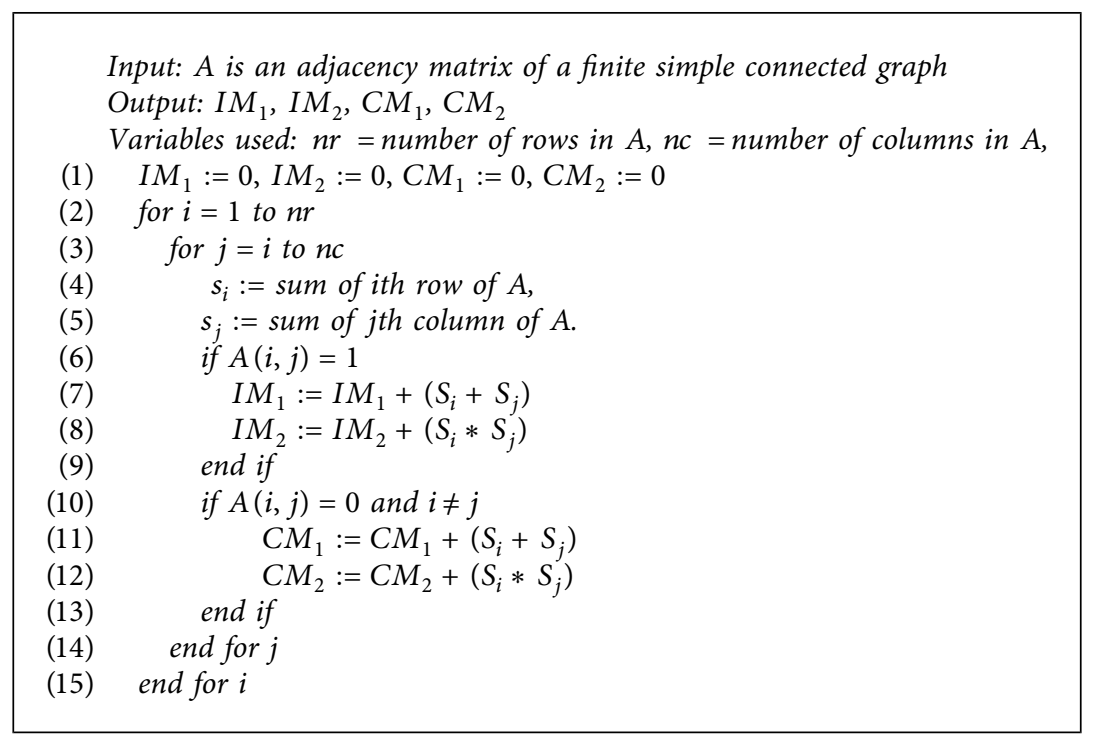

Algorithm 1: Computing the first Zagreb index $I M_{1}$, second Zagreb index $I M_{2}$, first Zagreb coindex $C M_{1}$, and second Zagreb coindex $\mathrm{CM}_{2}$.

$$
\begin{aligned}
& A=\left[\begin{array}{llll}
0 & 1 & 0 & 0 \\
1 & 0 & 1 & 0 \\
0 & 1 & 0 & 1 \\
0 & 0 & 1 & 0
\end{array}\right], \\
& B=\left[\begin{array}{llllllll}
0 & 1 & 0 & 0 & 1 & 1 & 0 & 0 \\
1 & 0 & 1 & 0 & 1 & 1 & 1 & 0 \\
0 & 1 & 0 & 1 & 0 & 1 & 1 & 1 \\
0 & 0 & 1 & 0 & 0 & 0 & 1 & 1 \\
1 & 1 & 0 & 0 & 0 & 1 & 0 & 0 \\
1 & 1 & 1 & 0 & 1 & 0 & 1 & 0 \\
0 & 1 & 1 & 1 & 0 & 1 & 0 & 1 \\
0 & 0 & 1 & 1 & 0 & 0 & 1 & 0
\end{array}\right], \\
& C=\left\{\begin{array}{llllllllllllllll}
0 & 1 & 0 & 0 & 1 & 1 & 0 & 0 & 1 & 1 & 0 & 0 & 1 & 0 & 0 & 0 \\
1 & 0 & 1 & 0 & 1 & 1 & 1 & 0 & 1 & 1 & 1 & 0 & 0 & 0 & 0 & 0 \\
0 & 1 & 0 & 1 & 0 & 1 & 1 & 1 & 0 & 1 & 1 & 1 & 0 & 0 & 0 & 0 \\
0 & 0 & 1 & 0 & 0 & 0 & 1 & 1 & 0 & 0 & 1 & 1 & 0 & 0 & 0 & 0 \\
1 & 1 & 0 & 0 & 0 & 1 & 0 & 0 & 1 & 0 & 0 & 0 & 1 & 1 & 0 & 0 \\
1 & 1 & 1 & 0 & 1 & 0 & 1 & 0 & 0 & 0 & 0 & 0 & 1 & 1 & 1 & 0 \\
0 & 1 & 1 & 1 & 0 & 1 & 0 & 1 & 0 & 0 & 0 & 0 & 0 & 1 & 1 & 1 \\
0 & 0 & 1 & 1 & 0 & 0 & 1 & 0 & 0 & 0 & 0 & 0 & 0 & 0 & 1 & 1 \\
1 & 1 & 0 & 0 & 1 & 0 & 0 & 0 & 0 & 1 & 0 & 0 & 1 & 1 & 0 & 0 \\
1 & 1 & 1 & 0 & 0 & 0 & 0 & 0 & 1 & 0 & 1 & 0 & 1 & 1 & 1 & 0 \\
0 & 1 & 1 & 1 & 0 & 0 & 0 & 0 & 0 & 1 & 0 & 1 & 0 & 1 & 1 & 1 \\
0 & 0 & 1 & 1 & 0 & 0 & 0 & 0 & 0 & 0 & 1 & 0 & 0 & 0 & 1 & 1 \\
1 & 0 & 0 & 0 & 1 & 1 & 0 & 0 & 1 & 1 & 0 & 0 & 0 & 1 & 0 & 0 \\
0 & 0 & 0 & 0 & 1 & 1 & 1 & 0 & 1 & 1 & 1 & 0 & 1 & 0 & 1 & 0 \\
0 & 0 & 0 & 0 & 0 & 1 & 1 & 1 & 0 & 1 & 1 & 1 & 0 & 1 & 0 & 1 \\
0 & 0 & 0 & 0 & 0 & 0 & 1 & 1 & 0 & 0 & 1 & 1 & 0 & 0 & 1 & 0
\end{array}\right\} .
\end{aligned}
$$




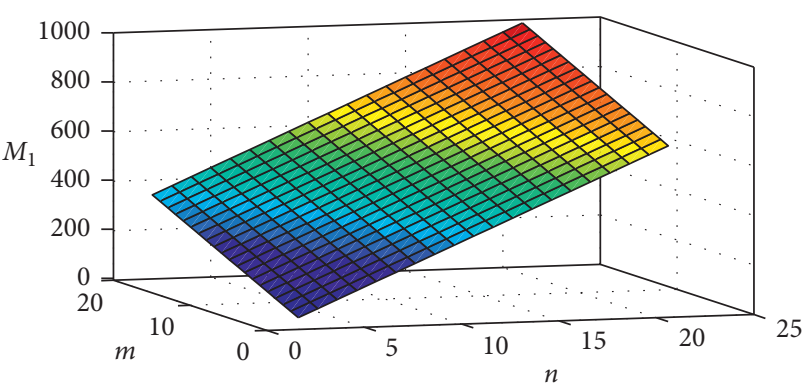

(a)

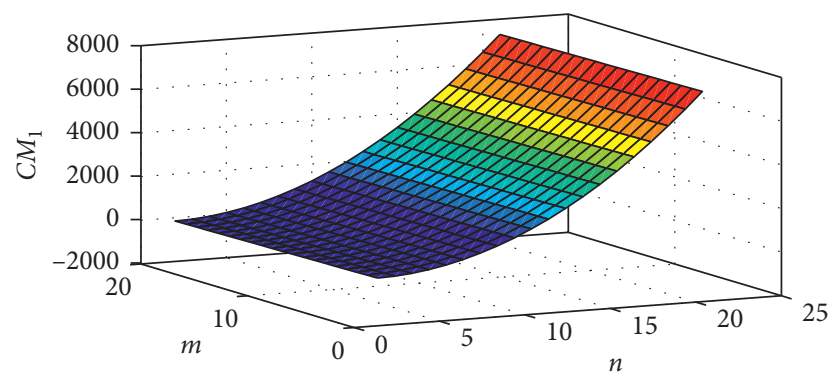

(c)

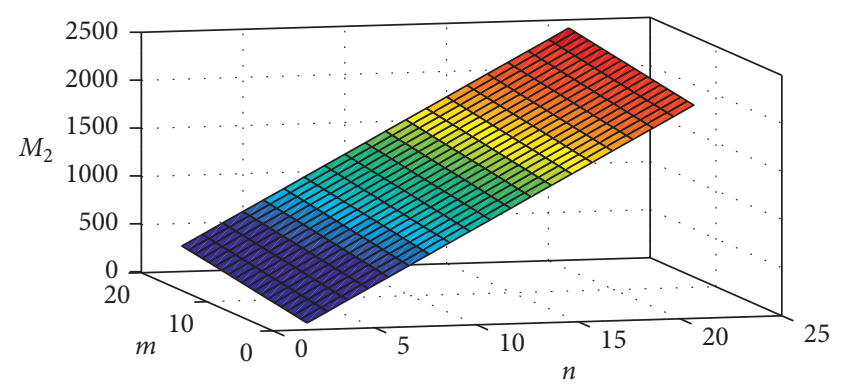

(b)

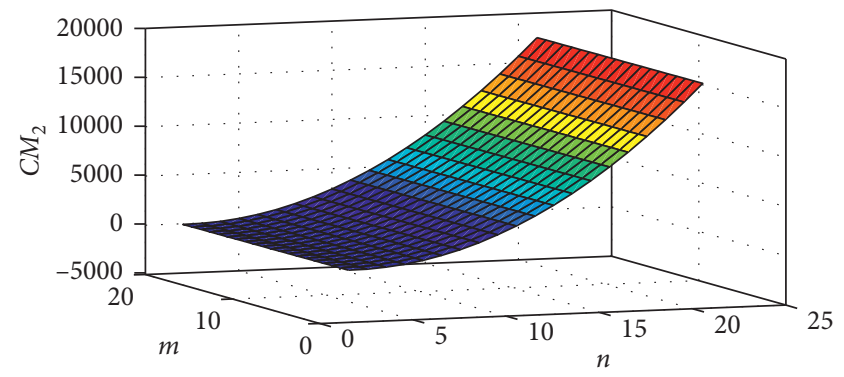

(d)

Figure 3: 3D surface plot of Zagreb indices and coindices of $S D\left(P_{n}\right)$.

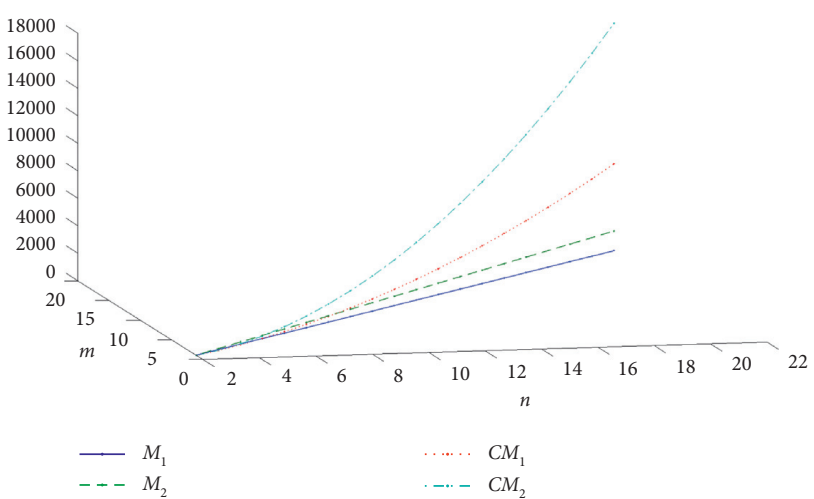

FIgURE 4: 3D curve plot of Zagreb indices and coindices of $S D\left(P_{n}\right)$.

The result of the algorithm is given as follows:

$\begin{array}{llllll}\text { Graph } & \text { Matrix } & A M_{1} & A M_{2} & C M_{1} & C M_{2} \\ P_{4} & A & 10 & 8 & 8 & 5 \\ S D^{1 *}\left(P_{4}\right) & B & 136 & 288 & 88 & 156 \\ S D^{2 *}\left(P_{4}\right) & C & 756 & 2652 & 864 & 2802\end{array}$.

Now, we provide graphs of Zagreb indices and coindices. Such type of graphical representation will be more helpful to study the dynamics of topological descriptors of the molecular graphs. Here, we present the strong double graph of the path graph, $S D\left(P_{n}\right)$, where $2 \leq n \leq 21$ and $m=n-1$. In Figure 3, the behaviour of the first Zagreb index $M_{1}$ and second Zagreb index $M_{2}$ is linear as the straight plane, and the behaviour of the first Zagreb coindex $C M_{1}$ and second Zagreb coindex $\mathrm{CM}_{2}$ is nonlinear as the curved form. In
Figure 4, we have drawn the curves of these indices and coindices for $S D\left(P_{n}\right)$ to understand their dynamics. This figure shows the relation between Zagreb indices and coindices as $M_{1} \leq M_{2} \leq C M_{1} \leq C M_{2}$.

\section{Conclusion}

We have presented generalized explicit formulas to calculate the first Zagreb index $M_{1}(G)$, second Zagreb index $M_{2}(G)$, first Zagreb coindex $\bar{M}_{1}(G)$, and second Zagreb coindex $\bar{M}_{2}(G)$ of the strong double graph $S D(G)$ and $k$-iterated strong double graph $S D^{k *}(G)$. The relation between these indices and coindices is also presented as $M_{1} \leq M_{2} \leq C M_{1} \leq C M_{2}$. We have also presented an algorithm with a given adjacency matrix to verify these indices and coindices by programming and numerically. Computergenerated graphs are also given to understand the dynamics of these indices and coindices.

This family of graphs can be considered for other degreebased and distance-related topological indices for further studies.

\section{Data Availability}

No data were used to support this study.

\section{Conflicts of Interest}

The authors declare that there are no conflicts of interest.

\section{Authors' Contributions}

All authors contributed equally to this work. 


\section{References}

[1] B. Danail and D. H. Rouvray, Chemical Graph Theory: Introduction and Fundamentals, CRC Press, Boca Raton, FL, USA, 1991.

[2] R. Todeschini and V. Consonni, Handbook of Molecular Descriptors, Wiley-VCH, Weinheim, Germany, 2000.

[3] R. Todeschini and V. Consonni, "New local vertex invariants and molecular descriptors based on functions of the vertex degrees," MATCH Communications in Mathematical and in Computer Chemistry, vol. 64, pp. 359-372, 2010.

[4] H. H. Lowell and K. B. Lemont, Molecular Connectivity in Chemistry and Drug Research, Academic Press, Cambridge, MA, USA, 1976.

[5] H. Wiener, "Structural determination of paraffin boiling points," Journal of the American Chemical Society, vol. 69, no. 1, pp. 17-20, 1947.

[6] A. R. Ashrafi, T. Došlić, and A. Hamzeh, "The Zagreb coindices of graph operations," Discrete Applied Mathematics, vol. 158, no. 15, pp. 1571-1578, 2010.

[7] L. Yang, X. Ai, and L. Zhang, "The Zagreb coindices of a type of composite graph," Hacettepe Journal of Mathematics and Statistics, vol. 45, pp. 1135-1142, 2016.

[8] I. Gutman and N. Trinajstić, "Graph theory and molecular orbitals. Total $\varphi$-electron energy of alternant hydrocarbons," Chemical Physics Letters, vol. 17, no. 4, pp. 535-538, 1972.

[9] S. Nikolic, G. Kovacevic, A. Milicevic, and N. Trinajstic, "The Zagreb indices 30 years after," Croatica Chemica Acta, vol. 76, pp. 113-124, 2003.

[10] I. Gutman, "Multiplicative Zagreb indices of trees," Bulletin of the Society of Mathematicians Banja Luka, vol. 18, pp. 17-23, 2011.

[11] M. Eliasi, A. Iranmanesh, and I. Gutman, "Multiplicative versions of first Zagreb index," MATCH Communications in Mathematical and in Computer Chemistry, vol. 68, pp. 217230, 2012.

[12] K. Xu and K. C. Das, "Trees, unicyclic, and bicyclic graphs extremal with respect to multiplicative sum Zagreb index," MATCH Communications in Mathematical and in Computer Chemistry, vol. 68, pp. 257-272, 2012.

[13] K. C. Das, "On comparing Zagreb indices of graphs," MATCH Communications in Mathematical and in Computer Chemistry, vol. 63, pp. 433-440, 2010.

[14] T. Doslic, "Vertex-weighted wiener polynomials for composite graphs," Ars Mathematica Contemporanea, vol. 1, pp. 66-80, 2008.

[15] A. R. Ashrafi, T. Doslic, and A. Hamzeh, "Extremal graphs with respect to the Zagreb coindices," MATCH Communications in Mathematical and in Computer Chemistry, vol. 65, pp. 85-92, 2011. 